\title{
Ejaculatio praecox und erektile Dysfunktion können kombiniert behandelt werden
}

\begin{abstract}
_ Dass Ejaculaio praecox (EP) und erektile Dysfunktion (ED) gemeinsam auftreten, ist angesichts der hohen Prävalenz - jeweils rund $20 \%$ - keine Seltenheit.

Serotonin ist der wichtigste inhibitorische Neurotransmitter in der Kontrolle der Ejakulation, erläuterte Prof. Chris McMahon, Sydney, Australien. Daher erscheint es logisch, hier anzusetzen, wenn die Ejakulation zu früh erfolgt. Dapoxetin (Priligy ${ }^{\circledR}$ ), das die Serotonin-Wiederaufnahme hemmt, eigne sich aufgrund seiner kurzen Halbwertzeit und schnellen Anflutung besonders gut für
\end{abstract}

eine bedarfsgesteuerte Therapie der EP, so McMahon.

In einer aktuellen, noch unveröffentlichten Studie unter der Leitung von McMahon erhielten 495 Männer, die bereits wegen Potenzstörungen einen PDE-5-Inhibitor bekamen und über EP klagten, zusätzlich bedarfsweise entweder 30 oder 60 mg Dapoxetin oder Placebo.

Schon in früheren Studien hatte sich gezeigt, dass Dapoxetin die intravaginale Ejakulationslatenzzeit ab der ersten Dosis signifikant verlängerte ( $p<0,001$ gegen Place- bo). Das bestätigte sich auch hier: Schon ab der ersten Woche zeigte sich ein signifikanter Unterschied zwischen den beiden Gruppen $(p<0,001)$, der bei Studienende nach zwölf Wochen fast zwei Minuten betrug (Ejakulationslatenzzeit 3,4 Minuten unter Placebo, 5,1 Minuten unter Dapoxetin). Parallel dazu verbesserten sich die Ergebnisse in den Domänen "Ejakulationskontrolle", "Persönlicher Distress" und "Zufriedenheit" des Premature Ejaculation Profile.

Manuela Arand

Satellitensymposium „Premature Ejaculation and Men's/Couple's Sexual Health", im Rahmen des Kongresses der International and European Societies for Sexual Medicine, Berlin, 22. Juni 2013, Veranstalter: Berlin-Chemie, Berlin

\section{Firmenjubiläum: 150 Jahre Bayer}

— Schrecksekunde für den Arzt Dr. Dimitris Voliotis. Während einer Konferenz zu den Studien zum Krebsmittel Nexavar ${ }^{\circledR}$ in einem New Yorker Hotel sagt ein Sprecher des Prüfkomitees: „Meine Damen und Herren, wir müssen die Testreihe stoppen." Doch schnell folgt die Erlösung. „Die Ergebnisse sind positiv.“ Das Medikament soll so schnell wie möglich allen Patienten zugänglich gemacht werden. Diesen Tag werde er nie vergessen, berichtete der Leiter der Klinischen Entwicklung für Krebsmedikamente bei Bayer in dem Buch „Das Erfinder-Unternehmen", das der Leverkusener Konzern anlässlich des 150-jährigen Jubiläums herausgegeben hat. „Man muss sich vorstellen, dass der Druck vieler Monate, sogar Jahre, die Erwartung, die Anspannung, die Skepsis, die Zweifel in einem Moment von einem abfallen", sagte der Arzt, der in Köln Medizin

\section{Zoledronsäure neu im Portfolio}

— medac hat seine Produktpalette der Bisphosphonate erweitert. Zusätzlich zu Pamifos (Pamidronat) steht in Deutschland seit Mai Zoledronsäure medac als Konzentrat $(4 \mathrm{mg} / 5 \mathrm{ml})$ und jetzt auch als fertige Infusionslösung (4 mg/100 ml) zur Verfügung.

Zoledronsäure medac ist zur Prävention skelettbezogener Komplikationen bei erwachsenen Patienten mit fortgeschrittenen auf das Skelett ausgedehnten Tumorerkrankungen zugelassen.

medac bietet sowohl das Konzentrat als auch die fertige Infusionslösung in den Packungsgrößen 1x1 (N1), 4x1 (N2) und 1x1 (KP) an. Die Infusionslösung bedarf keiner Zubereitung, sondern kann unmittelbar appliziert werden. Zudem ist die Flasche der Infusionslösung mit einem Bügeletikett versehen und kann direkt am Infusionsständer angehängt werden. Beide Darreichungsformen werden als 15-minütige Infusion gegeben.

Nach Informationen von medac, Wedel studiert hat und heute im US-Bundesstaat New Jersey lebt. Die Gewissheit, mit neuen Medikamenten Menschen helfen zu können, sei für ihn jeden Tag aufs Neue ein Ansporn, so Voliotis. Nexavar ${ }^{\circledR}$, das 2005 auf den Markt gekommen ist, ist eine der vielen Innovationen im Arzneimittelbereich, die zum Erfolg und zur weltweiten Bekanntheit von Bayer beigetragen haben. An erster Stelle steht natürlich Aspirin ${ }^{\circledast}$ aus dem Jahr 1899 - mit den Worten von Bayer-Vorstandschef Dr. Marijn Dekkers „ein echter Weltstar". Jetzt ruhen große Hoffnungen auf dem Gerinnungshemmer Xarelto ${ }^{\circledR}$ aus dem Jahr 2008.

Auf den Erfolgen dürfe sich Bayer nicht ausruhen. „Im Gegenteil, wir müssen in den kommenden Jahren noch einen Zahn zulegen", mahnte Dekker. Gefordert sind nach seiner Einschätzung nicht nur neue Moleküle und Produkte, sondern innovative Prozesse, kreative Ideen, bessere Dienstleistungen. Dafür sei die Innovationskultur von zentraler Bedeutung.

Bundeskanzlerin Angela Merkel (CDU) macht sich um die Zukunft von Bayer - „ein Symbol für den Innovations- und High TechStandort Deutschland" - offenbar keine Sorgen. „Ein Unternehmen, das auf eine 150-jährige Geschichte blicken kann, blickt auch weit in die Zukunft", sagte sie. Der Name Bayer habe einen festen Platz in der deutschen Industriegeschichte und stehe für den Erfolg. Ilse Schlingensiepen

Festakt „150 Jahre Bayer“, Köln, 16. Juli 2013 Veranstalter: Bayer, Leverkusen 